\title{
The pathwise numerical approximation of stationary solutions of semilinear stochastic evolution equations*
}

\author{
T. Caraballo \\ Dpto. de Ecuaciones Diferenciales y Análisis Numérico, \\ Universidad de Sevilla, Apdo. de Correos 1160 \\ 41080 Sevilla, Spain \\ E-mail: caraball@us.es \\ and \\ P.E. Kloeden \\ Fachbereich Mathematik, Johann Wolfgang Goethe Universität \\ D-60054 Frankfurt am Main, Germany \\ E-mail: kloeden@math.uni-frankfurt.de
}

December 13, 2005

\begin{abstract}
Under a one-sided dissipative Lipschitz condition on its drift, a stochastic evolution equation with additive noise of the reaction-diffusion type is shown to have a unique stochastic stationary solution which pathwise attracts all other solutions. A similar situation holds for each Galerkin approximation and each implicit Euler scheme applied to these Galerkin approximations. Moreover, the stationary solution of the Euler schemes converges pathwise to that of the Galerkin system as the stepsize tends to zero and the stationary solutions of the Galerkin systems converge pathwise to that of the evolution equation as the dimension increases. The analysis is carried out on random partial and ordinary differential equations obtained from their stochastic counterparts by substraction of appropriate Ornstein-Uhlenbeck stationary solutions.
\end{abstract}

*Partially supported by the Ministerio de Ciencia y Tecnología (Spain) and FEDER (European Community) grant BFM2002-03068. Peter Kloeden was also supported by the Ministerio de Educación y Ciencia (Spain) under the grant SAB2004-0146, within the Programa de Movilidad del Profesorado universitario español y extranjero. 
AMS Subject Classification: Primary 60H35, Secondary 60H15, 60H25

Key words: stochastic partial differential equations, random partial and ordinary differential equations, Galerkin approximations, implicit Euler scheme, stationary solutions, Ornstein-Uhlenbeck solution.

\section{Introduction}

The pathwise approximation of stationary solutions of semilinear stochastic evolution equations through both spatial discretization in the form of Galerkin stochastic differential equations (SDEs) and temporal discretization in the form of implicit Euler approximations of the Galerkin SDEs is the main interest of this paper. A typical motivating example is the noisy reaction-diffusion equation with additive noise

$$
\frac{\partial u}{\partial t}=\Delta u+f(u)+\eta_{t}
$$

reformulated as a semilinear parabolic stochastic partial differential equation (SPDE)

$$
d U_{t}=[\Delta U+f(U)] d t+d W_{t},
$$

on a bounded domain $\mathcal{O}$ in $\mathbb{R}^{d}$ with sufficiently smooth boundary $\partial \mathcal{O}$ and a Dirichlet boundary condition, where $\eta_{t}$ is a Gaussian white noise corresponding to a scalar space-time Wiener process and $f: \mathbb{R}^{1} \rightarrow \mathbb{R}^{1}$ satisfies a one-sided dissipative Lipschitz condition and is sufficiently regular to ensure that the above system has a strong as well as a weak (variational) solution on each finite time interval [7, 12].

Da Prato and Zabczyk [8], section 11, have shown the existence of a unique asymptotically stable invariant measure for such equations. Here we will focus on the pathwise dynamics and establish the existence of unique asymptotically stable stationary solution of each of the SPDE, its Galerkin SDE approximations and their implicit Euler schemes and their pathwise convergence as the dimension of the Galerkin approximation increases and the Euler step size decreases. In order to obtain pathwise estimates we will first convert these into random PDE, ODE and difference equations with linear transformations in which appropriate Ornstein-Uhlenbeck stationary stochastic processes are subtracted.

The paper is structured as follows: In the next section we formulate the stochastic evolution equations to be considered in a functional analytical setting. In section 3 we briefly review basic ideas and results from the theory of random dynamical systems and 
then in section 4 we informally state our main result, which is proved in the remaining sections of the paper. We prove the existence and uniqueness of a stochastic stationary solution successively for the random PDE, then its random Galerkin ODE and then for their implicit Euler schemes. Finally in section 8 we prove the pathwise convergence of these stationary solutions, first those of the Galerkin systems to that of the random PDE as the dimension increases, and then of the stationary solutions the numerical schemes to that of corresponding Galerkin system as the time step tends to zero. These results then transfer back to the original Ito stochastic setting by addition of the appropriate Ornstein-Uhlenbeck processes.

\section{Semilinear stochastic evolution equations}

We will consider a general setup involving Hilbert-valued Wiener process which contains the motivating example as a special case. Let $H$ be a separable Hilbert space $H$ and let $W$ be an $H$-valued Wiener process on a filtered probability space $\left(\Omega, \mathcal{F},\left\{\mathcal{F}_{t}\right\}_{t \in \mathbb{R}}, \mathbb{P}\right)$ with a symmetric covariance operator $Q$ of trace class. In addition, we assume that there exists a Gelfand triplet $V \subset H \subset V^{\prime}$ of separable Hilbert spaces, where $V^{\prime}$ denotes the dual of $V$ (see Temam [25] page 55 for more details) and $a_{1}>0$ is the constant of the injection $V \subset H$, i.e.

$$
a_{1}|u|^{2} \leq\|u\|^{2}, \quad \text { for } v \in V,
$$

where $|\cdot|$ and $\|\cdot\|$ denote the norms of $H$ and $V$, respectively, $(\cdot, \cdot)$ denotes the inner product in $H$ and $\langle\cdot, \cdot\rangle$ denote the duality mapping between $V^{\prime}$ and $V$.

Let $-A: V \rightarrow V^{\prime}$ be a positive, linear and continuous operator for which there exists $\alpha>0$ such that

$$
\langle-A u, u\rangle \geq \alpha\|u\|^{2}, \quad \text { for all } u \in V,
$$

and a sequence of eigenvalues $\lambda_{j}$ and corresponding eigenfunctions $\phi_{j} \in V$, that is with

$$
-A \phi_{j}=\lambda_{j} \phi_{j}, \quad j=1,2, \ldots,
$$

such that they form an orthonormal basis in $H$ with $\lambda_{j} \rightarrow \infty$ monotonically as $j \rightarrow \infty$ (see [7] for operators satisfying this condition). We note that each $u \in H$ has the unique representation in terms of the eigenfunctions of the operator $-A$, specifically, $u=\sum_{j=1}^{\infty} u_{j} \phi_{j}$

with norm $|u|=\sqrt{\sum_{j=1}^{\infty} u_{j}^{2}}$. Similarly, $\|u\|_{V}=\sqrt{\sum_{j=1}^{\infty} \lambda_{j} u_{j}^{2}}$ for $u \in V$. In addition, we note that condition (1) ensures that

$$
\|u\|_{V}^{2}=\langle-A u, u\rangle, \quad u \in V
$$

defines a norm which is equivalent to the original one in $V$, which we will use in the remaining of the paper. Then, it is well known (see, for instance, Dautray and Lions [9]) that $A$ is the generator of a strongly continuous semigroup $\left\{S_{A}(t)\right\}_{t \geq 0}$ in $H$ satisfying that

$$
\left\|S_{A}(t)\right\|_{\mathcal{L}(H)} \leq e^{-a t},
$$


where $a=\lambda_{1} \alpha>0$.

Finally, we assume that the nonlinear operator $f: H \rightarrow H$ is sufficient regular (in particular, at least continuous) and satisfies a one-sided Lipschitz condition:

$$
(u-v, f(u)-f(v)) \leq L|u-v|^{2}, \quad u, v \in H,
$$

for some $L \in \mathbb{R}$. (Typically, $f$ will be formed from a corresponding mapping of $\mathbb{R}$ into itself with similar properties.)

We will investigate stochastic evolution equations on $H$ of the form

$$
d U_{t}=A U_{t} d t+f\left(U_{t}\right) d t+d W_{t}, \quad U_{0}=u_{0},
$$

with an initial random variable $u_{0}$ which is $\left(\mathcal{F}_{0}, \mathcal{B}(H)\right)$-measurable.

Our motivating example can be set in this abstract formulation by simply taking $H=$ $L^{2}(\mathcal{O})$ and $V=H_{0}^{1}(\mathcal{O})$, where $H_{0}^{1}(\mathcal{O})$ is the Sobolev space of functions $u: \mathcal{O} \mapsto \mathbb{R}^{1}$ which vanish on $\partial \mathcal{O}$ such that $u$ and its first order generalized derivatives $D u$ belong to $L_{2}(\mathcal{O})$ with the norm $\|\cdot\|$.

There are a number of well known existence and uniqueness theorems (e.g. Pardoux [19], Krylov \& Rozovskii [18]) for weak (i.e. variational and understood in the sense of distributions) solution $U \in I^{2}(0, T ; V) \cap L^{2}(\Omega ; C(0, T ; H))$ of (5) for any $T \geq 0$ and initial value $u_{0} \in L^{2}\left(\Omega, \mathcal{F}_{0}, \mathbb{P}, H\right)$, which is also a mild solution and satisfies

$$
\mathbb{E} \sup _{t \in[0, T]}\|U(t)\|^{2}<\infty,
$$

for any $T>0$, where $I^{2}(0, T ; V)$ is the closed subspace of $L^{2}(\Omega \times(0, T), \mathcal{F} \otimes \mathcal{B}([0, T]), \mathbb{P} \otimes \mathrm{d} t ; V)$ of all stochastic processes which are $\mathcal{F}_{t}$-adapted for almost every $t$ in $(0, T)$. For this it is typically assumed that $f$ satisfies a linear growth bound or some kind of dissipativity conditon.

\section{Random dynamical systems}

We first recall some background notation and results. Let $(\Omega, \mathcal{F}, \mathbb{P})$ be a probability space and let $\mathcal{X}$ be a Hilbert space

Following Arnold [1] a random dynamical system (RDS) $(\theta, \phi)$ on $\Omega \times \mathcal{X}$ consists of a metric dynamical system $\theta$ on $\Omega$ and a cocycle mapping $\phi: \mathbb{R}^{+} \times \Omega \times \mathcal{X} \rightarrow \mathcal{X}$. Essentially (and sufficient for our purposes here), $\theta$ represents the driving noise process and $\phi$ the 
state space evolution of the system in $\mathcal{X}$. For example, for a stochastic differential equation on the Hilbert space $\mathcal{X}$ with a two-sided Hilbert valued Wiener process $W_{t}$, i.e. defined for all $t \in \mathbb{R}, \theta$ is defined by $\theta_{t} \omega(\cdot)=\omega(t+\cdot)-\omega(\cdot)$ on a canonical sample space $\Omega=$ $C_{0}(\mathbb{R}, \mathcal{X})=\{w \in C(\mathbb{R}, \mathcal{X}): \omega(0)=0\}$ and $\phi$ is defined by $\phi\left(t, \omega, x_{0}\right)=X_{t}^{x_{0}}(\omega)$, the solution of the SDE starting at $X_{0}^{x_{0}}(\omega)=x_{0}$. See $[1,16]$ for more details.

A family $\widehat{\mathcal{A}}=\{\mathcal{A}(\omega), \omega \in \Omega\}$ of nonempty measurable compact subsets $\mathcal{A}(\omega)$ of $\mathcal{X}$ is called $\phi$-invariant if $\phi(t, \omega, \mathcal{A}(\omega))=\mathcal{A}\left(\theta_{t} \omega\right)$ for all $t \geq 0$ and is called a random attractor if in addition it is pathwise pullback attracting in the sense that

$$
H_{\mathcal{X}}^{*}\left(\phi\left(t, \theta_{-t} \omega, D\left(\theta_{-t} \omega\right)\right), \mathcal{A}(\omega)\right) \rightarrow 0 \quad \text { as } \quad t \rightarrow-\infty
$$

for all suitable (i.e. in a given attracting universe [16]) families of $\widehat{D}=\{D(\omega), \omega \in \Omega\}$ of nonempty measurable bounded subsets $D(\omega)$ of $\mathcal{X}$. Here $H_{\mathcal{X}}^{*}$ is the Hausdorff semi-distance on $\mathcal{X}$. The following result ensures the existence of a random attractor (see, e.g., Crauel et al. [6], Schmalfuss [23]).

Theorem 1 Let $(\theta, \phi)$ be an $R D S$ on $\Omega \times \mathcal{X}$. If there exists a family $\widehat{B}=\{B(\omega), \omega \in \Omega\}$ of nonempty measurable compact subsets $B(\omega)$ of $\mathcal{X}$ and a $T_{\widehat{D}, \omega} \geq 0$ such that

$$
\phi\left(t, \theta_{-t} \omega, D\left(\theta_{-t} \omega\right)\right) \subset B(\omega), \quad \forall t \geq T_{\widehat{D}, \omega}
$$

for all families $\widehat{D}=\{D(\omega), \omega \in \Omega\}$ in the given attracting universe, then the $R D S(\theta, \phi)$ has a random attractor $\widehat{\mathcal{A}}=\{\mathcal{A}(\omega), \omega \in \Omega\}$ with the component subsets defined for each $\omega \in \Omega$ by

$$
\mathcal{A}(\omega)=\bigcap_{s>0} \overline{\bigcup_{t \geq s} \phi\left(t, \theta_{-t} \omega, B\left(\theta_{-t} \omega\right)\right)} .
$$

Alternatively, the sets $B(\omega)$ need only be closed and bounded provided the cocycle operator $\phi$ is compact.

Note that if the random attractor consists of singleton sets, i.e $\mathcal{A}(\omega)=\left\{X^{*}(\omega)\right\}$ for some random variable $X^{*}$, then $X_{t}^{*}(\omega):=X^{*}\left(\theta_{t} \omega\right)$ ) is a stationary stochastic process (see, e.g., [3] for more details).

It is not known if a general SPDE generates an RDS, but our special case with additive noise does (see [2] and [5] for similar situations on this field). In this case, the probability space $\Omega$ will be the canonical sample space of the infinite dimensional Wiener process, i.e. $C_{0}(\mathbb{R}, H)$ and $\theta_{t}$ will be the shift operator as defined above. 


\section{Main result: Existence of stationary solutions and the pathwise convergence of their approximations}

To ensure the existence of a unique stationary solution for the SPDE as well as for each of its approximating systems, we need some additional properties of the function $f$ in the SPDE, cf. Da Prato and Zabczyk [8].

1) $f$ satisfies a polynomial growth bound, i.e. there is a positive integer $p$ such that for all $u \in H$

$$
|f(u)| \leq K\left(1+|u|^{p}\right) .
$$

Hence there exists $m_{0}>0$ such that

$$
\int_{-\infty}^{t} e^{m s}|f(u(s))|^{2} d s<+\infty
$$

for any $m \in\left(0, m_{0}\right]$, and any continuous function $u: \mathbb{R} \rightarrow H$ with sub-exponential growth.

2) the constant $L$ in the one-sided Lipschitz condition (4) of $f$ satisfies

$$
\alpha \lambda_{1}-L>0
$$

Let $\mathcal{X}_{N}$ be the $N$-dimensional subspace of $V$ spanned by the $\left\{\phi_{1}, \cdots, \phi_{N}\right\}$ and let $P_{N}$ denote the projection of $H$ or $V$ onto $\mathcal{X}_{N}$. Write $U^{N}$ synonomously for $\left(U^{N, 1}, \cdots, U^{N, N}\right)^{T}$ $\in \mathbb{R}^{N}$ and $\sum_{j=1}^{N} U^{N, j} \phi_{j} \in \mathcal{X}_{N}$ according to context and define $A_{N}=\left.P_{N} A\right|_{\mathcal{X}_{N}}, W_{t}^{N}=P_{N} W_{t}$ and $f_{N}=\left.P_{N} f\right|_{\mathcal{X}_{N}}$. Since the probability space $\Omega$ under consideration is the canonical sample space of our infinite dimensional Wiener process, i.e. $C_{0}(\mathbb{R}, H)$, when we project onto a finite dimensional subspace $\mathcal{X}_{N}$ we use $W^{N}=P_{N} W$ instead of $W$, i.e., essentially just the first $N$ components of $\omega$ in $\Omega$. We can handle this by extending the projector $P_{N}$ to another operator $Q_{N}$ defined on $\Omega$ by

$$
Q_{N} \omega(\cdot)=\left\{P_{N} \omega(t), \quad t \in \mathbb{R}\right\} .
$$

Then, $Q_{N}$ and $\theta_{t}$ commute.

We now state our main result in a somewhat informal way, leaving a more formal formulation to the reader once the various necessary definitions have been presented in the sequel.

Theorem 2 Under the above setup and assumptions the SPDE, its Galerkin approximation in $\mathcal{X}_{N}$ and their implicit Euler approximations for sufficiently small time step $\Delta$ each has a unique stochastic stationary solution, denoted respectively by

$$
\bar{U}_{t}(\omega)=\bar{U}_{0}\left(\theta_{t} \omega\right), \quad \bar{U}_{t}^{N}(\omega)=\bar{U}_{0}^{N}\left(\theta_{t} \omega\right), \quad \bar{U}_{k}^{N, \Delta}(\omega)=\bar{U}_{0}^{N, \Delta}\left(\theta_{k \Delta} \omega\right),
$$


which pathwise attract all other solutions of their respective systems. Moreoever,

$$
\bar{U}_{0}^{N, \Delta}(\omega) \rightarrow \bar{U}_{0}^{N}(\omega) \quad \text { as } \Delta \rightarrow 0
$$

and

$$
\bar{U}_{0}^{N}(\omega) \rightarrow \bar{U}_{0}^{N}(\omega) \quad \text { as } \quad N \rightarrow \infty
$$

for each $\omega$.

We will first prove the existence and uniqueness of the stochastic stationary solutions for each the SPDE, Galerkin SDE and implicit Euler schemes in turn in the following consecutive sections. In order to obtain pathwise estimates we will first convert these into random PDE, ODE and difference equations. The pathwise convergences will be proved in the final section.

\section{SPDE and random PDE}

It follows from the one-sided Lipschitz condition (4) and assumption (8) that all solutions of the SPDE (5) converge to each other. To see this, notice that, due to the additive nature of the noise, we can subtract any two (strong or variational) solutions $U_{t}$ and $V_{t}$, giving

$$
d\left(U_{t}-V_{t}\right)=\left\{A\left(U_{t}-V_{t}\right)+f\left(U_{t}\right)-f\left(V_{t}\right)\right\} d t
$$

which means that the sample paths of $U_{t}-V_{t}$ are in fact differentiable with respect to time and satisfy pathwise the deterministic parabolic PDE

$$
\frac{\partial}{\partial t}\left(U_{t}-V_{t}\right)=A\left(U_{t}-V_{t}\right)+f\left(U_{t}\right)-f\left(V_{t}\right) .
$$

Then we have

$$
\begin{aligned}
\frac{1}{2} \frac{d}{d t}\left|U_{t}-V_{t}\right|^{2} & =\left\langle U_{t}-V_{t}, A\left(U_{t}-V_{t}\right)\right\rangle+\left(U_{t}-V_{t}, f\left(U_{t}\right)-f\left(V_{t}\right)\right) \\
& \leq-\alpha\left\|U_{t}-V_{t}\right\|^{2}+L\left|U_{t}-V_{t}\right|^{2} \leq-\left(\alpha \lambda_{1}-L\right)\left|U_{t}-V_{t}\right|^{2},
\end{aligned}
$$

from which it follows that

$$
\frac{d}{d t}\left|U_{t}-V_{t}\right|^{2} \leq-2\left(\alpha \lambda_{1}-L\right)\left|U_{t}-V_{t}\right|^{2}
$$

and hence that

$$
\left|U_{t}-V_{t}\right| \leq e^{-\left(\alpha \lambda_{1}-L\right) t}\left|U_{0}-V_{0}\right| \rightarrow 0 \quad \text { as } t \rightarrow \infty .
$$

We will now show that the solutions converge pathwise to a unique stationary solution. For this it will be more convenient to rewrite the SPDE (5) as a random PDE. Recall that 
$S_{A}(t)$ denotes the strongly continuous semigroup operator generated by the operator $A$, thus informally $S_{A}(t)=e^{A t}$. First we observe that (see Da Prato and Zabczyk [8, page 56])

$$
\widehat{U}_{0}\left(\theta_{t} \omega\right) \equiv \widehat{U}_{t}(\omega):=\int_{-\infty}^{t} S_{A}(t-s) d W_{s}(\omega)
$$

is the unique stationary solution of the linear SPDE

$$
d U_{t}=A U_{t} d t+d W_{t} .
$$

Subtracting it from any solution $U_{t}$ of the SPDE (5) we see that the difference $X_{t}=U_{t}-\widehat{U}_{t}$ is pathwise differentiable in time and satisfies pathwise the random PDE

$$
\frac{\partial}{\partial t} X_{t}=A X_{t}+f\left(X_{t}+\widehat{U}_{t}\right)
$$

Thus we obtain

$$
\begin{aligned}
\frac{d}{d t}\left|X_{t}\right|^{2} & =2\left\langle A X_{t}, X_{t}\right\rangle+2\left(X_{t}, f\left(X_{t}+\widehat{U}_{t}\right)-f\left(\widehat{U}_{t}\right)\right)+2\left(X_{t}, f\left(\widehat{U}_{t}\right)\right) \\
& \leq-2 \alpha\left\|X_{t}\right\|^{2}+2 L\left|X_{t}\right|^{2}+2\left|X_{t}\right|\left|f\left(\widehat{U}_{t}\right)\right| .
\end{aligned}
$$

Since $\alpha \lambda_{1}-L>0$ we have

$$
\frac{d}{d t}\left|X_{t}\right|^{2} \leq-\left(\alpha \lambda_{1}-L\right)\left|X_{t}\right|^{2}+\frac{1}{\alpha \lambda_{1}-L}\left|f\left(\widehat{U}_{t}\right)\right|^{2}
$$

Integrating from $t_{0}$ to $t$ we have

$$
\left|X_{t}\right|^{2} \leq e^{-\left(\alpha \lambda_{1}-L\right)\left(t-t_{0}\right)}\left|X_{t_{0}}\right|^{2}+\frac{e^{-\left(\alpha \lambda_{1}-L\right) t}}{\alpha \lambda_{1}-L} \int_{t_{0}}^{t} e^{\left(\alpha \lambda_{1}-L\right) s}\left|f\left(\widehat{U}_{s}\right)\right|^{2} d s
$$

Taking pullback convergence as $t_{0} \rightarrow-\infty$ pathwise in $H$ we obtain

$$
\left|X_{t}\right|^{2} \leq 1+\frac{e^{-\left(\alpha \lambda_{1}-L\right) t}}{\alpha \lambda_{1}-L} \int_{-\infty}^{t} e^{\left(\alpha \lambda_{1}-L\right) s}\left|f\left(\widehat{U}_{s}\right)\right|^{2} d s
$$

for all $t \geq T_{D}(\omega)$ for appropriate initial conditions $X_{t_{0}}(\omega) \in D$, an arbitrary closed and bounded subset of $H$.

This means that the family of closed and bounded random balls $\left\{B\left[0, R_{0}(\omega)\right], \omega \in \Omega\right\}$ in $H$ centred on the origin with radius $R_{0}(\omega)$, where

$$
R_{0}(\omega)^{2}:=1+\frac{1}{\alpha \lambda_{1}-L} \int_{-\infty}^{0} e^{\left(\alpha \lambda_{1}-L\right) s}\left|f\left(\widehat{U}_{0}\left(\theta_{s} \omega\right)\right)\right|^{2} d s
$$

is an absorbing family of sets for the cocycle generated by the SPDE. Since operator $A$ has a sequence of eigenvalues satisfying (2), then (see Chueshov [4], Chapter 2) the random PDE has a random attractor which in fact consists of a single unique stationary solution in $H$, which we will denote by $\bar{X}_{t}$. Hence the SPDE has a unique stationary solution $\bar{U}_{t}=\bar{X}_{t}+\widehat{U}_{t}$, which pathwise attracts all other solutions. 


\section{Ito-Galerkin SDE and Galerkin RDE}

By similar arguments to those for the above SPDE a unique stationary solution $U_{t}^{N}$ also exists for the $N$-dimensional Ito-Galerkin SDE of the SPDE with the initial value $U_{0}^{N}=P_{N} U_{0}$, since $f_{N}$ satisfies the same assumptions as $f$. Moreover, this Ito-Galerkin SDE generates an $N$-dimensional random dynamical system. (See Hausenblas $[14,15]$, where finite difference and wavelet approximations leading to similar types of Ito SDE are also considered).

The $N$-dimensional Ito-Galerkin SDE corresponding to the SPDE (5) is given by

$$
d U_{t}^{N}=\left\{A_{N} U_{t}^{N}+f_{N}\left(U_{t}^{N}\right)\right\} d t+d W_{t}^{N},
$$

where $f_{N}\left(U^{N}\right)$ inherits the one-sided dissipative Lipschitz condition (4) of $f$, specifically

$$
\left\langle U^{N}-V^{N}, f_{N}\left(U^{N}\right)-f_{N}\left(V^{N}\right)\right\rangle_{\mathbb{R}^{N}} \leq L\left|U^{N}-V^{N}\right|_{\mathbb{R}^{N}}^{2}
$$

while the matrix $A_{N}$, which is a diagonal matrix with $j t h$ diagonal component $-\lambda_{j}$ for $j=$ $1, \ldots, N$, satisfies the dissipative bound

$$
\left\langle U^{N}, A_{N} U^{N}\right\rangle_{\mathbb{R}^{N}} \leq-\alpha \lambda_{1}\left|U^{N}\right|_{\mathbb{R}^{N}}^{2}
$$

for all $U^{N} \in \mathbb{R}^{N}$.

The Ito-Galerkin SDE (10) inherits the strong contractivity of the SPDE, which is proved in much the same way as for the SPDE above. As there we can show that the difference of any two solutions is pathwise differentiable and satisfies the differential inequality

$$
\frac{d}{d t}\left|U_{t}^{N}-V_{t}^{N}\right|_{\mathbb{R}^{N}} \leq-\left(\alpha \lambda_{1}-L\right)\left|U_{t}^{N}-V_{t}^{N}\right|_{\mathbb{R}^{N}}
$$

which means all solutions converge pathwise to each other.

We introduce the Ornstein-Uhlenbeck process

$$
\widehat{U}_{t}^{N}=e^{A_{N} t} \int_{-\infty}^{t} e^{-A_{N} s} d W_{s}^{N},
$$

which is the unique pathwise asymptotically stable stationary random solution of the SDE with linear drift term

$$
d X_{t}=A_{N} X_{t} d t+d W_{t}^{N}
$$

on $\mathbb{R}^{N}$.

The solutions paths of the Ito-Galerkin SDE (10) are generally not differentiable, so in order to use the one-sided dissipative Lipschitz condition (11) we consider the difference $X_{t}^{N}$ $=U_{t}^{N}-\widehat{U}_{t}^{N}$ where $\widehat{U}_{t}^{N}$ is the Ornstein-Uhlenbeck stationary process (12) satisfying the linear 
equations (13). We note that $\widehat{U}_{t}^{N}=P_{N} \widehat{U}_{t}$, so $\widehat{U}_{t}^{N}(\omega)$ converges to $\widehat{U}_{t}(\omega)$ for each $\omega$. This difference is pathwise differentiable since the paths are continuous and satisfy the ordinary differential equation

$$
\frac{d}{d t} X_{t}^{N}=A_{N} X_{t}^{N}+f_{N}\left(X_{t}^{N}+\widehat{U}_{t}^{N}\right)
$$

which is called a random differential equation (RDE).

It follows that

$$
\begin{aligned}
\frac{d}{d t}\left|X_{t}^{N}\right|_{\mathbb{R}^{N}}^{2}= & 2\left\langle X_{t}^{N}, A_{N} X_{t}^{N}\right\rangle_{\mathbb{R}^{N}}+2\left\langle X_{t}^{N}, f_{N}\left(X_{t}^{N}+\widehat{U}_{t}^{N}\right)-f_{N}\left(\widehat{U}_{t}^{N}\right)\right\rangle_{\mathbb{R}^{N}} \\
& +2\left\langle X_{t}^{N}, f_{N}\left(\widehat{U}_{t}^{N}\right)\right\rangle_{\mathbb{R}^{N}} \\
\leq & -2\left(\alpha \lambda_{1}-L\right)\left|X_{t}^{N}\right|_{\mathbb{R}^{N}}^{2}+2\left|X_{t}^{N}\right|_{\mathbb{R}^{N}}\left|f_{N}\left(\widehat{U}_{t}^{N}\right)\right|_{\mathbb{R}^{N}}
\end{aligned}
$$

and thus

$$
\frac{d}{d t}\left|X_{t}^{N}\right|_{\mathbb{R}^{N}}^{2} \leq-\left(\alpha \lambda_{1}-L\right)\left|X_{t}^{N}\right|_{\mathbb{R}^{N}}^{2}+\frac{1}{\alpha \lambda_{1}-L}\left|f_{N}\left(\widehat{U}_{t}^{N}\right)\right|_{\mathbb{R}^{N}}^{2}
$$

from which we obtain

$$
\left|X_{t}^{N}\right|_{\mathbb{R}^{N}}^{2} \leq\left|X_{t_{0}}^{N}\right|_{\mathbb{R}^{N}}^{2} e^{-\left(\alpha \lambda_{1}-L\right)\left(t-t_{0}\right)}+\frac{e^{-\left(\alpha \lambda_{1}-L\right) t}}{\alpha \lambda_{1}-L} \int_{t_{0}}^{t} e^{\left(\alpha \lambda_{1}-L\right) s}\left|f_{N}\left(\widehat{U}_{s}^{N}\right)\right|_{\mathbb{R}^{N}}^{2} d s
$$

Pathwise pullback convergence (i.e. as $t_{0} \rightarrow-\infty$ and $t$ fixed) gives pullback absorption (cf. Theorem 1)

$$
\left|X_{t}^{N}(\omega)\right|_{\mathbb{R}^{N}}^{2} \leq R^{N}\left(\theta_{t} \omega\right)^{2}:=1+\frac{e^{-\left(\alpha \lambda_{1}-L\right) t}}{\alpha \lambda_{1}-L} \int_{-\infty}^{t} e^{\left(\alpha \lambda_{1}-L\right) s}\left|f_{N}\left(\widehat{U}_{s}^{N}(\omega)\right)\right|_{\mathbb{R}^{N}}^{2} d s
$$

for all $t \geq T_{D}(\omega)$ for any bounded set $D$ containing the $\omega$ realization of the initial value. It is straightforward to check that the integrability of $f$ is transferred to $f_{N}$ due to the subexponential growth of $\widehat{U}_{s}^{N}$ and condition (7). Moreover, the integrals exist here due to the assumption (7) and the relationship between the OU processes $\widehat{U}_{t}^{N}$ and $\widehat{U}_{t}$.

Thus

$$
\left|X_{t}^{N}(\omega)\right|_{\mathbb{R}^{N}} \leq R^{N}\left(\theta_{t} \omega\right), \quad \forall t \geq T_{D}(\omega)
$$

which implies that this system has a random attractor $\widehat{\mathcal{A}}=\{\mathcal{A}(\omega), \omega \in \Omega\}$. The strong contractivity condition proved earlier means that this random attractor consists of singleton sets formed by a stationary random solution $\bar{X}_{t}^{N}$ of the Galerkin RDE (14). Hence the ItoGalerkin SDE (10) has a random attractor consisting of a stationary random solution $\bar{U}_{t}^{N}=$ $\bar{X}_{t}^{N}+\widehat{U}_{t}^{N}$. (It is interesting to compare the estimates used in this section with alternative 
types of estimates used by Robinson [21, 22].)

For later, we note from the definition (9) of the radius $R_{0}(\omega)$ of the absorbing balls for the random PDE that

$$
R^{N}(\omega) \leq R_{0}(\omega) \quad \text { for all } N, \omega .
$$

In particular, this means that the difference of the stationary solution of the random PDE and the random Galerkin systems satisfies

$$
\left|\bar{X}_{t}^{N}(\omega)-P_{N} \bar{X}_{t}(\omega)\right|_{\mathbb{R}^{N}} \leq 2 R_{0}\left(\theta_{t} \omega\right) \quad \text { for all } t, N, \omega
$$

\section{$7 \quad$ Implicit Euler scheme}

Rather than discretizing the Ito-Galerkin SDE (10), we will reformulate it as a random differential equation (RDE), i.e. pathwise an ordinary differential equation, which we will discretize. This will allow us to consider pathwise approximations rather than mean-square approximations as is typical for Ito SDE.

Let $X_{t}^{N}=U_{t}^{N}-\widehat{U}_{t}^{N}$, where $U_{t}^{N}$ is a solution of the Ito-Galerkin SDE (10) and $\widehat{U}_{t}^{N}$ the Ornstein-Uhlenbeck stationary process defined by (12). Then $X_{t}^{N}$ is pathwise differentiable and satisfies the RDE (14) which has an asymptotically stable stationary solution $\bar{X}_{t}^{N}=$ $\bar{U}_{t}^{N}-\widehat{U}_{t}^{N}$, where $\bar{U}_{t}^{N}$ is the asymptotically stable stationary solution of the Ito-Galerkin SDE (10).

The implicit Euler scheme with constant time-step $\Delta$ for the RDE (14) is given by

$$
Y_{k+1}^{N}=Y_{k}^{N}+\left(A_{N} Y_{k+1}^{N}+f_{N}\left(Y_{k+1}^{N}+\widehat{U}_{(k+1) \Delta}^{N}\right)\right) \Delta
$$

assuming for notational convenience that one starts at time $t_{0}=0$ (otherwise one has to evaluate the OU process as $\left.\widehat{U}_{t_{0}+(k+1) \Delta}^{N}\right)$. In fact, we would have obtained the same scheme if we had started with the implicit Euler scheme for the Ito-Galerkin SDE and then applied our OU change of coordinates.

This scheme is strongly contractive in the same sense of the random PDE and its Galerkin RDE. Let $Y_{k}^{N}$ and $Z_{k}^{N}$ be two numerical solutions. Then

$$
\begin{aligned}
Y_{k+1}^{N}-Z_{k+1}^{N}= & \left.Y_{k}^{N}-Z_{k}^{N}+A_{N}\left(Y_{k+1}^{N}\right)-Z_{k+1}^{N}\right) \Delta \\
& +\left(f_{N}\left(Y_{k+1}^{N}+\widehat{U}_{(k+1) \Delta}^{N}\right)-f_{N}\left(Z_{k+1}^{N}+\widehat{U}_{(k+1) \Delta}^{N}\right)\right) \Delta .
\end{aligned}
$$


Taking the inner product with $Y_{k+1}^{N}-Z_{k+1}^{N}$ gives

$$
\begin{gathered}
\left|Y_{k+1}^{N}-Z_{k+1}^{N}\right|^{2}=\left\langle Y_{k+1}^{N}-Z_{k+1}^{N}, Y_{k}^{N}-Z_{k}^{N}\right\rangle+\left\langle Y_{k+1}^{N}-Z_{k+1}^{N}, A_{N}\left(Y_{k+1}^{N}-Z_{k+1}^{N}\right)\right\rangle \Delta \\
\quad+\left\langle Y_{k+1}^{N}-Z_{k+1}^{N}, f_{N}\left(Y_{k+1}^{N}+\widehat{U}_{(k+1) \Delta}^{N}\right)-f_{N}\left(Z_{k+1}^{N}+\widehat{U}_{(k+1) \Delta}^{N}\right)\right\rangle \Delta \\
\leq\left|Y_{k+1}^{N}-Z_{k+1}^{N}\right|\left|Y_{k}^{N}-Z_{k}^{N}\right|-\left(\alpha \lambda_{1}-L\right)\left|Y_{k+1}^{N}-Z_{k+1}^{N}\right|^{2} \Delta
\end{gathered}
$$

which leads to

$$
\left|Y_{k+1}^{N}-Z_{k+1}^{N}\right| \leq\left(1+\left(\alpha \lambda_{1}-L\right) \Delta\right)^{-1}\left|Y_{k}^{N}-Z_{k}^{N}\right|
$$

and the strong pathwise contractivity follows.

Taking the inner product on both sides of the implicit Euler scheme (16) with $Y_{k+1}^{N}$ we obtain

$$
\begin{gathered}
\left|Y_{k+1}^{N}\right|^{2}=\left\langle Y_{k+1}^{N}, Y_{k}^{N}\right\rangle+\left\langle Y_{k+1}^{N}, A_{N} Y_{k+1}^{N}\right\rangle \Delta+\left\langle Y_{k+1}^{N}, f_{N}\left(Y_{k+1}^{N}+\widehat{U}_{(k+1) \Delta}^{N}\right)\right\rangle \Delta \\
\leq\left|Y_{k+1}^{N}\right|\left|Y_{k}^{N}\right|-\alpha \lambda_{1}\left|Y_{k+1}^{N}\right| \Delta+\left\langle Y_{k+1}^{N}, f_{N}\left(\widehat{U}_{(k+1) \Delta}^{N}\right)\right\rangle \Delta \\
\quad+\left\langle Y_{k+1}^{N}+\widehat{U}_{(k+1) \Delta}^{N}-\widehat{U}_{(k+1) \Delta}^{N}, f_{N}\left(Y_{k+1}^{N}+\widehat{U}_{(k+1) \Delta}^{N}\right)-f_{N}\left(\widehat{U}_{(k+1) \Delta}^{N}\right)\right\rangle \Delta \\
\leq\left|Y_{k+1}^{N}\right|\left|Y_{k}^{N}\right|-\left(\alpha \lambda_{1}-L\right)\left|Y_{k+1}^{N}\right| \Delta+\left|Y_{k+1}^{N}\right|\left|f_{N}\left(\widehat{U}_{(k+1) \Delta}^{N}\right)\right| \Delta .
\end{gathered}
$$

Simplifying, dividing by $\left|Y_{k+1}^{N}\right|$ and rearranging, we obtain

$$
\left|Y_{k+1}^{N}\right| \leq\left(1+\left(\alpha \lambda_{1}-L\right) \Delta\right)^{-1}\left|Y_{k}^{N}\right|+\left(1+\left(\alpha \lambda_{1}-L\right) \Delta\right)^{-1}\left|f_{N}\left(\widehat{U}_{(k+1) \Delta}^{N}\right)\right| \Delta
$$

which gives

$$
\left.\left|Y_{k}^{N}\right| \leq\left(1+\left(\alpha \lambda_{1}-L\right) \Delta\right)^{-\left(k-k_{0}\right)}\left|Y_{k_{0}}^{N}\right|+\sum_{j=k_{0}}^{k-1}\left(1+\left(\alpha \lambda_{1}-L\right) \Delta\right)^{-(k-j)} \mid f_{N}\left(\widehat{U}_{(j+1) \Delta}^{N}\right)\right) \mid \Delta,
$$

where all norms are the Euclidean norm in $\mathbb{R}^{N}$.

This means that the $Y_{k}^{N}$ are absorbed into a ball of radius

$$
R_{\Delta}^{N}\left(\theta_{k \Delta} \omega\right):=1+\sum_{j=-\infty}^{k-1}\left(1+\left(\alpha \lambda_{1}-L\right) \Delta\right)^{-(k-j)}\left|f_{N}\left(\widehat{U}_{(j+1) \Delta}^{N}(\omega)\right)\right|_{\mathbb{R}^{N}} \Delta
$$


in finite time depending on the initial conditions. Thus $Y_{k}^{N}$ are absorbed into a ball of radius $R_{\Delta}^{N}\left(\theta_{k \Delta}\right)$ centred on 0 , i.e so the numerical scheme has a random attractor in this ball. In view of the strong pathwise contractivity property, this random attractor consists of a single set formed by a stationary solution $\bar{Y}_{k}^{N, \Delta}$.

We then define $\bar{U}_{t}^{N, \Delta}=\bar{Y}_{k}^{N, \Delta}+\widehat{U}_{k \Delta}^{N}$ which is also a stochastic stationary process which we compared with that of the Galerkin approximation in Theorem 2.

For later, we note that from the definitions we have $\left|f_{N}\left(\widehat{U}_{t}^{N}(\omega)\right)\right|_{\mathbb{R}^{N}} \leq\left|f\left(\widehat{U}_{t}(\omega)\right)\right|$, so there exists a $\Delta^{*}>0$ such that

$$
R_{\Delta}^{N}(\omega) \leq R_{*}(\omega)
$$

for all $0<\Delta \leq \Delta^{*}$ and positive integers $N$, where

$$
R_{*}(\omega):=1+\int_{-\infty}^{0} e^{\frac{1}{2}\left(\alpha \lambda_{1}-L\right) s}\left|f\left(\widehat{U}_{s}(\omega)\right)\right| d s
$$

In particular, this means that the numerical approximatrions all lie in a common compact ball for sufficeintly small step sizes.

\section{Convergence of approximating stationary solutions}

Note that the estimates above for the Galerkin RDE and its implicit Euler scheme remain valid in the $H$-norm if it is assumed that the RDE solution $X_{t}^{N}$ and the iterates $X_{k}^{N}$ of the numerical scheme (16) with the initial value $X_{0}^{N}=P_{N} X_{0}$ are interpreted as belonging to $\mathcal{X}_{N}$ rather than $\mathbb{R}^{N}$.

Let us write the stationary solutions of the random (not stochastic) equations in terms of given random variables, i.e.

$$
\bar{X}_{t}(\omega)=\bar{X}\left(\theta_{t} \omega\right), \quad \bar{X}_{t}^{N}(\omega)=\bar{X}^{N}\left(\theta_{t} \omega\right), \quad \bar{Y}_{k}^{N, \Delta}(\omega)=\bar{Y}^{N, \Delta}\left(\theta_{k \Delta} \omega\right) .
$$

\subsection{The random PDE and random Galerkin ODE}

We can compare the stationary solution $\bar{X}_{t}$ of the random PDE directly with the stationary solution $\bar{X}_{t}^{N}$ of a Galerkin random ODE. First we notice that $P_{N} \bar{X}_{t}$ satisfies the the $N$ dimensional random ODE

$$
\frac{d}{d t} P_{N} \bar{X}_{t}=P_{N} A \bar{U}_{t}+P_{N} f\left(\bar{X}_{t}+\widehat{U}_{t}\right)
$$


while any solution of the $N$-dimensional Galerkin random DE satisfies

$$
\frac{d}{d t} X_{t}^{N}=A_{N} X_{t}^{N}+f_{N}\left(X_{t}^{N}+\widehat{U}_{t}^{N}\right)
$$

Subtracting and using the definitions of $A_{N}$ and $f_{N}$ we obtain the random ODE in $\mathbb{R}^{N}$

$$
\frac{d}{d t}\left(P_{N} \bar{X}_{t}-X_{t}^{N}\right)=A_{N}\left(P_{N} \bar{X}_{t}-X_{t}^{N}\right)+P_{N} f\left(\bar{X}_{t}+\widehat{U}_{t}\right)-f_{N}\left(X_{t}^{N}+\widehat{U}_{t}^{N}\right) .
$$

Applying the estimate of the operator $A_{N}$ inherited from $A$, the one-sided Lipschitz condition on $f$, and similar estimates as above we have pathwise

$$
\begin{aligned}
\frac{d}{d t}\left|P_{N} \bar{X}_{t}-X_{t}^{N}\right|_{\mathbb{R}^{N}}^{2} & =2\left\langle P_{N} \bar{X}_{t}-X_{t}^{N}, A_{N}\left(P_{N} \bar{X}_{t}-X_{t}^{N}\right)\right\rangle_{\mathbb{R}^{N}} \\
& \leq-\left(\alpha \lambda_{1}-L\right)\left|P_{N} \bar{X}_{t}-X_{t}^{N}\right|_{\mathbb{R}^{N}}^{2}+\frac{1}{\alpha \lambda_{1}-L}\left|P_{N} f\left(\bar{U}_{t}\right)-P_{N} f\left(P_{N} \bar{U}_{t}\right)\right|_{\mathbb{R}^{N}}^{2},
\end{aligned}
$$

where $\bar{U}_{t}=\bar{X}_{t}+\widehat{U}_{t}$ is the stationary solution of the SPDE. Hence

$$
\frac{d}{d t}\left|P_{N} \bar{X}_{t}-X_{t}^{N}\right|_{\mathbb{R}^{N}}^{2}+\left(\alpha \lambda_{1}-L\right)\left|P_{N}\left(\bar{X}_{t}-X_{t}^{N}\right)\right|_{\mathbb{R}^{N}}^{2} \leq \frac{1}{\alpha \lambda_{1}-L}\left|f\left(\bar{U}_{t}\right)-f\left(P_{N} \bar{U}_{t}\right)\right|^{2}
$$

Integrating we obtain pathwise

$$
\begin{aligned}
\left|P_{N}\left(\bar{X}_{t}-\bar{X}_{t}^{N}\right)\right|_{\mathbb{R}^{N}}^{2} \leq e^{-\gamma\left(t-t_{0}\right)}\left|P_{N}\left(\bar{X}_{t_{0}}-\bar{X}_{t_{0}}^{N}\right)\right|_{\mathbb{R}^{N}}^{2} & \\
& \quad+\frac{e^{-\gamma t}}{\alpha \lambda_{1}-L} \int_{t_{0}}^{t} e^{\gamma s}\left|f\left(\bar{U}_{s}\right)-f\left(P_{N} \bar{U}_{s}\right)\right|^{2} d s
\end{aligned}
$$

for any positive $\gamma \leq 2\left(\alpha \lambda_{1}-L\right)$, which means the integrals here exist pathwise and are bounded for all $t \geq t_{0}$.

Now the projection bound

$$
\left|\bar{U}_{s}(\omega)-P_{N} \bar{U}_{s}(\omega)\right|=\left|\left(I-P_{N}\right) \bar{U}_{s}(\omega)\right|^{2} \leq K\left(T_{0}, T_{1}, \omega\right) \lambda_{N+1}^{-1}
$$

holds uniformly in $s$ on any bounded interval $\left[T_{0}, T_{1}\right]$, so $\left|f\left(\bar{U}_{s}(\omega)\right)-f\left(P_{N} \bar{U}_{s}(\omega)\right)\right|$ converges to 0 as $N \rightarrow \infty$ uniformly in $s$ on any bounded interval $\left[T_{0}, T_{1}\right]$, which means that any integral

$$
\int_{T_{0}}^{T_{1}} e^{\gamma s}\left|f\left(\bar{U}_{s}(\omega)\right)-f\left(P_{N} \bar{U}_{s}(\omega)\right)\right|^{2} d s \rightarrow 0 \quad \text { as } \quad N \rightarrow \infty .
$$

Let $\omega$ be fixed and suppose that for some $T$ there is an $\epsilon_{0}>0$ and and a subsequence $N_{j} \rightarrow \infty$ for which

$$
\left|P_{N_{j}} \bar{X}_{T}(\omega)-\bar{X}_{T}^{N_{j}}(\omega)\right|_{\mathbb{R}^{N_{j}}}^{2} \geq \epsilon_{0} \text { for all } j
$$


Then by (18) for any $t_{0}<T$ we have

$$
\epsilon_{0} \leq e^{-\gamma\left(T-t_{0}\right)} \limsup _{j \rightarrow \infty}\left|P_{N_{j}} \bar{X}_{t_{0}}(\omega)-\bar{X}_{t_{0}}^{N_{j}}(\omega)\right|_{\mathbb{R}^{N_{j}}}^{2}
$$

But by (15) we have

$$
\left|P_{N_{j}} \bar{X}_{t_{0}}(\omega)-\bar{X}_{t_{0}}^{N_{j}}(\omega)\right|_{\mathbb{R}^{N_{j}}}^{2} \leq 4 R_{0}\left(\theta_{t_{0}} \omega\right)^{2}
$$

uniformly in $j$, which means that

$$
\epsilon_{0} e^{\gamma\left(T-t_{0}\right)} \leq 4 R_{0}\left(\theta_{t_{0}} \omega\right)^{2}
$$

from which we conclude that

$$
R_{0}\left(\theta_{t_{0}} \omega\right) \rightarrow \infty \quad \text { as } t_{0} \rightarrow-\infty
$$

However, this is not possible from the definition (9), the polynomial growth bound on $f$ and the subexponential growth of the OU stationary solutions.

Thus we must have the convergence

$$
\left|P_{N} \bar{X}_{t_{0}}(\omega)-\bar{X}_{t_{0}}^{N}(\omega)\right|_{\mathbb{R}^{N}}^{2} \rightarrow 0 \quad \text { as } N \rightarrow \infty
$$

which we will combine with a projection bound on $\left(I-P_{N}\right) \bar{X}_{t_{0}}(\omega)$ to obtain the desired pathwise convergence

$$
\left|\bar{X}_{0}(\omega)-\bar{X}_{0}^{N}(\omega)\right| \rightarrow 0 \quad \text { as } \quad N \rightarrow \infty
$$

for any $\omega$.

\subsection{Galerkin RDE and its implicit Euler scheme}

Fix $N$. We can prove that $\bar{X}^{N, \Delta}(\omega)$ converges pathwise to $\bar{X}^{N}(\omega)$ as $\Delta \rightarrow 0$ using a proof by contradiction following the Section 5 of [16] using the pathwise error bounds in [10, 13], compare with $[21,22]$ which gives convergence in expectation only.

Suppose that there is an $\omega$, sequence $\Delta_{j} \rightarrow 0$ as $j \rightarrow \infty$ and an $\epsilon_{0}>0$ such that

$$
\left|\bar{X}^{N, \Delta_{j}}(\omega)-\bar{X}^{N}(\omega)\right| \geq \epsilon_{0}
$$

In Section 6 we saw that $\bar{X}^{N, \Delta_{j}}(\omega)$ belong to a common compact ball $B^{N}(\omega)$ of radius $R_{*}(\omega)$ defined by (17) each $\omega$. Now the family of balls $\left\{B^{N}(\omega), \omega\right\}$ is pullback attracted to the Galerkin RDE stationary solution. Thus there is a $T(\omega)$ such that

$$
\left|\phi^{N}\left(t, \theta_{-t} \omega, X^{N}\left(\theta_{-t} \omega\right)\right)-\bar{X}^{N}(\omega)\right| \leq \epsilon_{0} / 4
$$


for $t \geq T(\omega)$ and any $X^{N}\left(\theta_{-t} \omega\right) \in B^{N}\left(\theta_{-t} \omega\right)$. Let $k_{j}$ be the first integer so that $k_{j} \Delta_{j} \geq$ $T(\omega)$. Applying this to $\bar{X}^{N, \Delta_{j}}\left(\theta_{-k_{j} \Delta_{j}} \omega\right) \in B^{N}\left(\theta_{-k_{j} \Delta_{j}} \omega\right)$ we then have

$$
\left|\phi^{N}\left(k_{j} \Delta_{j}, \theta_{-k_{j} \Delta_{j}} \omega, \bar{X}^{N, \Delta_{j}}\left(\theta_{-k_{j} \Delta_{j}} \omega\right)\right)-\bar{X}^{N}(\omega)\right| \leq \epsilon_{0} / 4
$$

We then use the pathwise global discretization error of the implicit Euler scheme for the RDE (see [13]) of the interval $[-1-T(\omega), 0]$ to obtain

$$
\left|Y_{k_{j}}^{N, \Delta_{j}}\left(\theta_{-k_{j} \Delta_{j}} \omega\right)-\phi^{N}\left(k_{j} \Delta_{j}, \theta_{-k_{j} \Delta_{j}} \omega, \bar{X}^{N, \Delta_{j}}\left(\theta_{-k_{j} \Delta_{j}} \omega\right)\right)\right| \leq \epsilon_{0} / 4
$$

for $j$ large enough, where $Y_{k_{j}}^{N, \Delta_{j}}\left(\theta_{-k_{j} \Delta_{j}} \omega\right)$ is the implicit Euler solution with step size $\Delta_{j}$ starting at $\bar{X}^{N, \Delta_{j}}\left(\theta_{-k_{j} \Delta_{j}} \omega\right)$. Since this is the stationary solution, we have $Y_{k_{j}}^{N, \Delta_{j}}\left(\theta_{-k_{j} \Delta_{j}} \omega\right)=$ $\bar{X}^{N, \Delta_{j}}(\omega)$, and hence

$$
\left|\bar{X}^{N, \Delta_{j}}(\omega)-\phi^{N}\left(k_{j} \Delta_{j}, \theta_{-k_{j} \Delta_{j}} \omega, \bar{X}^{N, \Delta_{j}}\left(\theta_{-k_{j} \Delta_{j}} \omega\right)\right)\right| \leq \epsilon_{0} / 4
$$

We combine this with the preceding estimate to obtain

$$
\left|X^{N, \Delta_{j}}(\omega)-\bar{X}^{N}(\omega)\right| \leq \epsilon_{0} / 2
$$

which contradicts (19).

Thus we have pathwise convergence of the numerical stationary solution to that of the Galerkin RDE.

Finally, we note that the order of convergence here may be less than that of the same scheme for vector fields which are smooth in $t$ due to the fact that the sample paths of the OU process $\widehat{U}_{t}$ are only Hölder continuous rather than differentiable [10, 13].

\section{References}

[1] L. Arnold, Random Dynamical Systems, Springer-Verlag, Berlin, 1997.

[2] T. Caraballo, I.D. Chueshov and J.A. Langa, Existence of invariant manifolds for coupled parabolic and hyperbolic stochastic partial differential equations, Nonlinearity 18 (2005), 747-767.

[3] T. Caraballo, P.E. Kloeden and B. Schmalfuß, Exponentially stable stationary solutions for evolutions equations and their perturbation, Appl. Math. Optim. 50 (2004), 183-207.

[4] I.D. Chueshov, Introduction to the Theory of Infinite-Dimensional Dissipative Systems, Acta Scientific Publishing House, Kharkov, 1999. 
[5] I.D. Chueshov and M. Scheutzow, Inertial manifolds and forms for stochastically perturbed retarded semilinear parabolic equations, J. Dyn. Diff. Eqns. 13 (2001), 355-380.

[6] H. Crauel, A. Debussche, and F. Flandoli, Random attractors, J. Dyn. Diff. Eqns. 9 (1997), 307-341.

[7] G. Da Prato and G. Zabczyk, Stochastic Equations in Infinite Dimensions, Cambridge University Press, Cambridge, 1992.

[8] G. Da Prato and G. Zabczyk, Ergodicity for Infinite Dimensional Systems, London Mathematical Society, Lecture Note Series Vol. 229, Cambridge University Press, Cambridge, 1996.

[9] R. Dautray and J.L. Lions, Mathematical Analysis and Numerical Methods for Science and Technology: Vol. 3, Spectral Theory and Applications, Springer-Verlag, Berlin, 1990.

[10] I. Gyöngy, A note on Euler's approximations, Potential Anal. 8 (1998), 205-216.

[11] W. Grecksch and P.E. Kloeden, Time-discretized Galerkin approximations of parabolic stochastic PDEs, Bulletin Austral. Math. Soc. 54 (1996), 79-84.

[12] W. Grecksch and C. Tudor, Stochastic Evolution Equations, Akademie- Verlag, Berlin, 1995.

[13] L. Grüne and P.E. Kloeden, Pathwise approximation of random ordinary differential equations, BIT 41 (2001), 710-721.

[14] E. Hausenblas, Numerical analysis of semilinear stochastic evolution equations in Banach spaces, J. Computat. Appl. Math. 147 (2002), 485-516.

[15] E. Hausenblas, Approximation of semilinear stochastic evolution equations. Potential Anal. 18 (2003), 141-186.

[16] P.E. Kloeden, H. Keller and B. Schmalfuss, Towards a theory of random numerical dynamics, in Stochastic Dynamics. Editors: H. Crauel and V. M. Gundlach, SpringerVerlag, 1999, pp. 259-282.

[17] P.E. Kloeden and E. Platen, Numerical Solution of Stochastic Differential Equations, Springer-Verlag, Berlin, 1992.

[18] N.V. Krylov and B.L. Rozovskii, Ob evoljucionnych stochastičeskich uravneniach, volume 14 of Sovremennych problemy matematiki, pages 71-146. Moskva, 1979.

[19] E. Pardoux, Équations aux dérivées partielles stochastiques non linéaires monotones. Étude de solutions fortes de type Ito, PhD These, Université Paris Sud, 1975. 
[20] X. Mao, Stochastic Differential Equations and their Applications, Horwood Publishing, Chichester, 1997.

[21] J.C. Robinson, Stability of random attractors under perturbation and approximation, J. Diff. Eqns. 186 (2002), 652-669.

[22] J.C. Robinson, Stability of random attractors for a backwards Euler scheme, Stochastics G Dynamics 4 (2004), 175-184.

[23] B. Schmalfuss, Backward cocycles and attractors of stochastic differential equations, in International Seminar on Applied Mathematics-Nonlinear Dynamics: Attractor Approximation and Global Behaviour. Editors: V. Reitmann, T. Riedrich and N. Koksch, pages 185-192, 1992.

[24] A.M. Stuart and A.R. Humphries, Dynamical Systems and Numerical Analysis, Cambridge University Press, Cambridge, 1996.

[25] R. Temam, Infinite-Dimensional Dynamical Systems in Mechanics and Physics. Springer-Verlag, Berlin, second edition, 1997. 